\title{
ON THE CRITICAL STATES OF A BINARY SYSTEM
}

\section{BY PAUL SAUREL}

The conditions which are satisfied when a system of two phases formed from two independent components reaches a critical state were given by Gibbs in his memoir "On the Equilibrium of Heterogeneous Substances." Of the two conditions given by Gibbs only the first has been used by subsequent writers, and yet some of the most interesting of the theorems relating to the critical state are consequences of the second condition. These theorems, however, are well known, but heretofore they have been obtained as consequences of hypotheses which are less simple than those of Gibbs. In the present note we shall give a simple demonstration of Gibbs' conditions and we shall then show their importance in the theory of the critical state.

In what follows we shall suppose that the systems considered have been formed from two independent components. Moreover, by the term concentration of a phase we shall understand the mass of the first component which is present in the unit of mass of the phase.

The fundamental assumption of Gibbs may be stated as follows: For any given pair of components there exists an analytical function $\xi$ of the variables $T, \Pi$ and $x$, such that if $T, \Pi$ and $x$ denote the temperature, the pressure and the concentration of a homogeneous phase in equilibrium, $\zeta$ is equal to the thermodynamic potential of the unit of mass of that phase. This function is, in general, many-valued. Thus, for any given values of $\mathrm{T}$ and $\Pi$, we may represent the relation between $\zeta$ and $x$ by a number of continuous curves in the $\zeta x$ plane. To each branch of the $\zeta$ function there corresponds a $\zeta$ curve. ${ }^{x}$

It can be shown that, when a homogeneous phase is in

${ }^{1}$ On the Equilibrium of Heterogeneous Substances, p. I78. 
equilibrium, its specific entropy $\eta$, its specific volume $v$ and what for convenience we shall call its chemical potential $\mu$, are given by the equations

$$
-\eta=\frac{\partial \zeta}{\partial T}, \quad v=\frac{\partial \zeta}{\partial \Pi}, \quad \mu=\frac{\partial \zeta}{\partial x} .
$$

Moreover, when the homogeneous phase is in stable equilibrium, we must have, for any virtual change which leaves the temperature and the pressure unchanged,

$$
\delta \zeta>\mu \delta x
$$

in which the variations are to be construed strictly. ${ }^{\mathrm{x}}$ But, on the other hand, it follows, from the definition of $\zeta$, that in passing from one state of equilibrium to an adjacent state of equilibrium at the same temperature and under the same pressure, we must have

$$
\delta \zeta=\frac{\partial \zeta}{\partial x} \delta x+\frac{1}{2} \frac{\partial^{2} \zeta}{\partial x^{2}} \delta x^{2}+\cdots,
$$

in which the omitted terms are of the third and higher degrees in $\delta x$. From $I, 2$ and 3 it follows at once that when a homogeneous phase is in stable equilibrium at a given temperature and under a given pressure,

$$
\frac{\partial^{2} \zeta}{\partial x^{2}}>0
$$

Thus at every point which corresponds to a phase in stable equilibrium the $\zeta$ curve is convex toward the $x$ axis.

If the system consists of two phases in equilibrium it can be shown that

$$
\begin{gathered}
\left(\frac{\partial \zeta}{\partial x}\right)_{1}=\left(\frac{\partial \zeta}{\partial x}\right)_{2} \\
\zeta_{1}-x_{1}\left(\frac{\partial \zeta}{\partial x}\right)_{1}=\zeta_{2}-x_{2}\left(\frac{\partial \zeta}{\partial x}\right)_{2}
\end{gathered}
$$

in which the subscripts I and 2 refer to the two phases. The geometrical interpretation of these conditions is that the tangents at the points I and 2 coincide. Moreover, it can be shown

${ }^{1}$ On the Equilibrium of Heterogeneous Substances, p. I66. 
that if the system is in stable equilibrium, condition 4 is satisfied at each of the points $I$ and 2 .

The points I and 2 may belong to the same or to different $\zeta$ curves. By varying the temperature and the pressure it may be possible to change the relative position and the shape of the $\zeta$ curves so that finally the points $\mathrm{I}$ and 2 are brought into coincidence. If the points which are thus made to coincide belong to different $\zeta$ curves we reach an indifferent state of the system $;^{x}$ if, on the other hand, the two points which are brought.into coincidence belong to the same $\zeta$ curve we reach a critical state of the system. ${ }^{2}$ In the first case, it is easy to see that, although $x_{x}$ and $x_{2}$ become equal, $\eta_{x}$ and $\eta_{2}, v_{x}$ and $v_{2}$ do not. For $-\eta_{x}$ and $v_{x}$ are equal to the derivatives with respect to $T$ and $\Pi$ of that branch of the $\zeta$ function which corresponds to the first $\zeta$ curve, while $-\eta_{2}$ and $v_{2}$ are equal to the derivatives with respect to $T$ and $\Pi$ of that branch of the $\zeta$ function which corresponds to the second $\zeta$ curve. In the second case, however, not only $x_{1}$ and $x_{2}$ become equal, but also $\eta_{1}$ and $\eta_{2}, v_{1}$ and $v_{z}$.

Let us consider the second case. If we remember that at each of the points of contact of the double tangent the curve is convex toward the $x$ axis, it follows that a line parallel to the double tangent and slightly above it cuts the curve in four points. Accordingly, if the points $\mathrm{I}$ and 2 be made to coincide, the tangent to the curve at the limiting point will have with the curve a contact of the third order. The conditions for this are well known; they are

$$
\frac{\partial^{2} \zeta}{\partial x^{2}}=0, \quad \frac{\partial^{3} \zeta}{\partial x^{3}}=0 .
$$

These are Gibbs' conditions. ${ }^{3}$

Before discussing these conditions let us return to the consideration of equations 5 and 6 . As these equations give two conditions between the four quantities $T, \Pi, x_{1}$ and $x_{2}$, it follows that two of these quantities, $T$ and $\Pi$, for example, can be chosen

${ }^{1}$ On the Equilibrium of Heterogeneous Substances, p. 183.

2 van Rijn van Alkemade. Zeit. phys. Chem. Ir, 298 (1893).

${ }^{3}$ On the Equilibrium of Heterogeneous Substances, p. 191. 
arbitrarily. Accordingly, if we take three rectangular axes in space along which we shall measure $T, \Pi$ and $x$, the values of $x_{x}$ and $x_{2}$ which correspond to different pairs of values of $T$ and II will determine two surfaces which we shall call the concentration surfaces.

Equations 7 give us two relations between the three quantities $T, \Pi$ and $x$. Accordingly, the points in the $T \Pi x$ space which correspond to critical states of the system, all lie on a curve. This curve may, with propriety, be called the critical line. Moreover, from the manner in which the critical states have been defined, it follows that the critical line is common to both of the concentration surfaces.

The equations which connect the simultaneous changes in $\mathrm{T}, \Pi$ and $x$ as we pass from point to point on the concentration surfaces are due to van der Waals. ${ }^{\mathrm{I}}$ He has shown that they can be written in the following simple form:

$$
\begin{aligned}
& v_{21} d \Pi=\eta_{21} d \mathrm{~T}+\left(x_{2}-x_{1}\right)\left(\frac{\partial^{2} \zeta}{\partial x^{2}}\right)_{1} d x_{1}, \\
& v_{12} d \Pi=\eta_{12} d \mathrm{~T}+\left(x_{1}-x_{2}\right)\left(\frac{\partial^{2} \xi}{\partial x^{2}}\right)_{2} d x_{2},
\end{aligned}
$$

in which

$$
\begin{aligned}
& v_{21}=v_{2}-v_{1}-\left(x_{2}-x_{1}\right)\left(\frac{\partial v}{\partial x}\right)_{1}, \\
& v_{12}=v_{1}-v_{2}-\left(x_{1}-x_{2}\right)\left(\frac{\partial v}{\partial x}\right)_{2}, \\
& \eta_{21}=\eta_{2}-\eta_{1}-\left(x_{2}-x_{1}\right)\left(\frac{\partial \eta}{\partial x}\right)_{1} \\
& \eta_{12}=\eta_{1}-\eta_{2}-\left(x_{1}-x_{2}\right)\left(\frac{\partial \eta}{\partial x}\right)_{2} .
\end{aligned}
$$

If we suppose that the system has reached an indifferent state we have

$$
x_{1}=x_{2}, \quad \eta_{1} \neq \eta_{2}, \quad v_{1} \neq v_{2} .
$$

${ }^{1}$ Archives néerlandaises, 24, I5 (1890). Zeit. phys. Chem. 5, I43 (1890). Archives néerlandaises, 30,267 (1896). Die Continuität des gasförmigen und flüssigen Zustandes, 2, 108, I I2, I 79 ( I900). 
Equations 8 and 9 both reduce to the form

$$
\left(v_{2}-v_{1}\right) d \Pi=\left(\eta_{2}-\eta_{1}\right) d \mathrm{~T} \text {. }
$$

Thus the points in the $T \Pi x$ space which correspond to the indifferent states of the system lie on a line which is common to the two concentration surfaces. This line may be called the indifferent line of the system. From equation ${ }_{5} 5$ it follows that along the indifferent line the two concentration surfaces have a common tangent plane and that this plane is constantly perpendicular to the $T \Pi$ plane. Further, the slope of the projection upon the $T \Pi$ plane of the indifferent line is given by equation $I_{5}$ or, in more familiar form, by the equation

$$
\frac{d \mathrm{II}}{d \mathrm{~T}}=\frac{\eta_{2}-\eta_{1}}{v_{2}-v_{1}}
$$

This extension of Clapeyron's equation to the indifferent states of a bivariant binary system is due to Gibbs. ${ }^{\mathrm{r}}$

Let us consider next the manner in which the two concentration surfaces meet along the critical line. At every point of the critical line we have

$$
\begin{gathered}
x_{1}=x_{2}, \quad \eta_{1}=\eta_{2}, \quad v_{1}=v_{2}, \\
\frac{\partial^{2} \zeta}{\partial x^{2}}=0, \quad \frac{\partial^{3} \zeta}{\partial x^{3}}=0 .
\end{gathered}
$$

The coefficient of every term in equations 8 and 9 is thus equal to zero. However, if we refer to equations IO, II, I2 and I3 and if we expand $v_{2}, v_{\mathrm{r}}, \eta_{2}$ and $\eta_{\mathrm{r}}$ by Taylor's theorem, we find that the following approximate relations hold:

$$
\begin{aligned}
& v_{21}=\frac{1}{2}\left(x_{2}-x_{1}\right)^{2}\left(\frac{\partial^{2} v}{\partial x^{2}}\right)_{1}, \\
& v_{12}=\frac{1}{2}\left(x_{1}-x_{2}\right)^{2}\left(\frac{\partial^{2} v}{\partial x^{2}}\right)_{2}, \\
& \eta_{21}=\frac{1}{2}\left(x_{2}-x_{1}\right)^{2}\left(\frac{\partial^{2} \eta}{\partial x^{2}}\right)_{1}, \\
& \eta_{12}=\frac{1}{2}\left(x_{1}-x_{2}\right)^{2}\left(\frac{\partial^{2} \eta}{\partial x^{2}}\right)_{2} .
\end{aligned}
$$

${ }^{1}$ On the Equilibrium of Heterogeneous Substances, p. I55. 
Equations 8 and 9 then take the form

$$
\begin{aligned}
& \left(\frac{\partial^{2} v}{\partial x^{2}}\right)_{1} d \Pi=\left(\frac{\partial^{2} \eta}{\partial x^{2}}\right)_{1} d \mathrm{~T}+\frac{2}{x_{2}-x_{1}}\left(\frac{\partial^{2} \zeta}{\partial x^{2}}\right)_{1} d x_{1}, \\
& \left(\frac{\partial^{2} v}{\partial x^{2}}\right)_{2} d \Pi=\left(\frac{\partial^{2} \eta}{\partial x^{2}}\right)_{2} d \mathrm{~T}+\frac{2}{x_{1}-x_{2}}\left(\frac{\partial^{2} \xi}{\partial x^{2}}\right)_{2} d x_{2} .
\end{aligned}
$$

To find the limiting value of the least term in equation 23 we observe that we have approximately

$$
\left(\frac{\partial^{2} \zeta}{\partial x^{2}}\right)_{1}=\frac{\partial^{2} \zeta}{\partial x^{2}}+\left(x_{1}-x\right) \frac{\partial^{3} \zeta}{\partial x^{3}}
$$

in which the point to which $x$ refers is any point on the $\zeta$ curve in the immediate neighborhood of the point $I$. If we suppose that the point to which $x$ refers is one of the two points on the $\zeta$ curve between $I$ and 2 for which

$$
\frac{\partial^{2} \zeta}{\partial x^{2}}=0
$$

the first term on the right-hand side of equation 25 disappears and the coefficient of $d x_{x}$ in equation 23 becomes

$$
2 \frac{x_{1}-x}{x_{2}-x_{1}} \frac{\partial^{3} \zeta}{\partial x^{3}} \text {. }
$$

Since $x-x_{x}$ is not larger than $x_{2}-x_{x}$ it follows that the limit of their ratio cannot be infinite. It follows then from the second of equations I 8 that the limit of expression 26 is zero. Accordingly, at the limit, equations 23 and 24 reduce to the same form, viz :

$$
\frac{\partial^{2} v}{\partial x^{2}} d \Pi=\frac{\partial^{2} \eta}{\partial x^{2}} d \mathrm{~T}
$$

Accordingly, along the critical line the two concentration surfaces have a common tangent plane, and this plane is constantly perpendicular to the $T \Pi$ plane.

From the fact that along the indifferent and the critical lines the tangent plane to the two concentration surfaces is perpendicular to the $T \Pi$ plane, we can derive at once several interesting theorems. In the first place, if the indifferent and the critical lines intersect, their projections upon the $T \Pi$ plane will 
be tangent to each other. In the second place, if the section of the concentration surfaces made by a plane parallel to the $T \Pi$ plane cut either the indifferent or the critical line, the projection of this curve upon the $T \Pi$ plane will be tangent to the projection of the indifferent or of the critical line. Finally, a section of the concentration surfaces by a plane parallel to the T $x$ plane or parallel to the $\Pi x$ plane has at the points in which it cuts the indifferent and the critical lines tangents which are parallel to the $x$ axis.

The theorems which we have deduced as consequences of Gibbs' conditions are well known. Demonstrations of them have been given by van der Waals and by Duhem. ${ }^{2}$

New York, July 9, 1902.

${ }^{1}$ Archives néerlandaises, 30, 266 (I896). Die Continuität des gasförmigen und flüssigen Zustandes, 2, I3I-I46 (Ig00).

2 Traité élémentaire de Mécanique chimique, 4, Iog-I21 ( I899). 